\title{
TRAUMATIC PENILE FRACTURE WITH BILATERAL CORPOREAL RUPTURE
}

\author{
Sorin Nedelea ${ }^{1}$, Iulian Slavu ${ }^{1}$, Andrei $\operatorname{Sin}^{2}$, Octavian Patrascanu ${ }^{2}$, Adrian Tulin ${ }^{1}$, Bogdan Socea ${ }^{3}$, \\ Alecu Lucian ${ }^{1}$
}

${ }^{1}$ Agrippa Ionescu Emergency Clinical Hospital, Bucharest, Romania

${ }^{2}$ Burghele Hospital, Urology Department, Bucharest, Romania

${ }^{3}$ Pantelimon Emergency Hospital Bucharest, Romania

\begin{tabular}{c}
\hline ORIGINAL \\
PAPER \\
\hline
\end{tabular}

Doi: $10.33695 /$ rojes.v2i2.26

Accepted: 15.12 .2020
Corresponding author: Iulian Slavu iulian.slavu@yahoo.com

\begin{abstract}
A 24-year-old man with no urological history presented to the emergency room for rapid onset severe penile pain, tenderness, swelling, and ecchymosis, which occurred during vaginal sexual intercourse. Clinical examination was suggestive of penile fracture with a high suspicion of bilateral corporeal involvement. Urethrography excluded an injury to the corpus spongiosum. Surgical exploration confirmed the bilateral laceration of the corpora cavernosa and tunica albuginea which was repaired with absorbable sutures. Penile fractures are true urological emergencies whose surgical treatment must not be delayed to prevent long-term sequelae. Bilateral corporeal rupture accounts for only $2-10 \%$ percent of penile fractures and usually involves the urethra, especially when the lacerations are situated ventrally.
\end{abstract}

Keywords: penile fracture, trauma, surgery

\section{Introduction}

A penile fracture represents an urogenital injury in which the tunica albuginea of the penis is torn and the corpus cavernosum is ruptured due to a traumatic injury. It is considered an emergency in urology, mainly because the delay of treatment can lead to several anatomical and sexual dysfunctions [1]. The rupture of the penis is rather uncommon, but several studies show an increasing rate over the years [2].

This injury is described only on an erect penis when a blunt trauma causes the rupture of the tunica albuginea of the corpora cavernosa [3]. During an erection, tunica albuginea thins to $0.25-0.5 \mathrm{~mm}$ and withstands intracavernous pressures of up to $1500 \mathrm{~mm} \mathrm{Hg}$ [2]. When this pressure is exceeded due to a traumatic event, the thinned tunica albuginea is sheared. Most commonly, the right corpus cavernosum is affected, but it can involve both corpora cavernosa and even the corpus spongiosum or penile urethra $[2,3]$.

The surgery for penile fracture should be performed as fast as possible due to the complications associated with delayed treatment. It involves opening the skin, evacuation of the hematoma, and closure of the defects of tunica albuginea. If the urethra is also affected, repair or other methods such as urethral catheterization or suprapubic bladder catheterization are necessary [2]. 
The present paper intends to highlight the steps of diagnosis and treatment of a penile fracture that involves both corpora cavernosa, without urethral injury.

\section{Case report}

An otherwise healthy 24-year-old male was admitted to the emergency room with severe penile pain, tenderness, and swelling with acute onset, two hours following blunt trauma during sexual intercourse. The trauma was followed by a 'snapping sound', immediate loss of erection, and severe pain. Physical examination revealed ventral and scrotal ecchymosis, tenderness, and swelling of the penis with ventral hematoma extending to the scrotum and perineum and what appeared to be two continuity defects on the ventral and lateral sides of both corpora, suggesting rupture of the tunica albuginea of the corpora cavernosa.

The patient did not present any voiding difficulty or hematuria. His initial evaluation included superficial ultrasound showing a focal tear in the ventral wall of both corpora cavernosa with important hematoma displacing the corpus spongiosum and the urethra. A retrograde urethrogram showed an uncompromised urethra without any extravasation of the contrast material. The patient underwent emergency surgical exploration with general anesthesia 2 hours after the admission. A subcoronal circumferential incision was made and degloving was performed. At first, the hematoma was evacuated, revealing a partial tear on the ventral side of the tunica albuginea of both corpora cavernosa (Figure 1-2).

A 16 Ch Foley Catheter was placed with no difficulties. A vessel loop was used to isolate the urethra. Interrupted 3/0 absorbable sutures were used to repair the rupture of both corporeal lacerations. The redundant foreskin was removed after regloving (Figure.3).

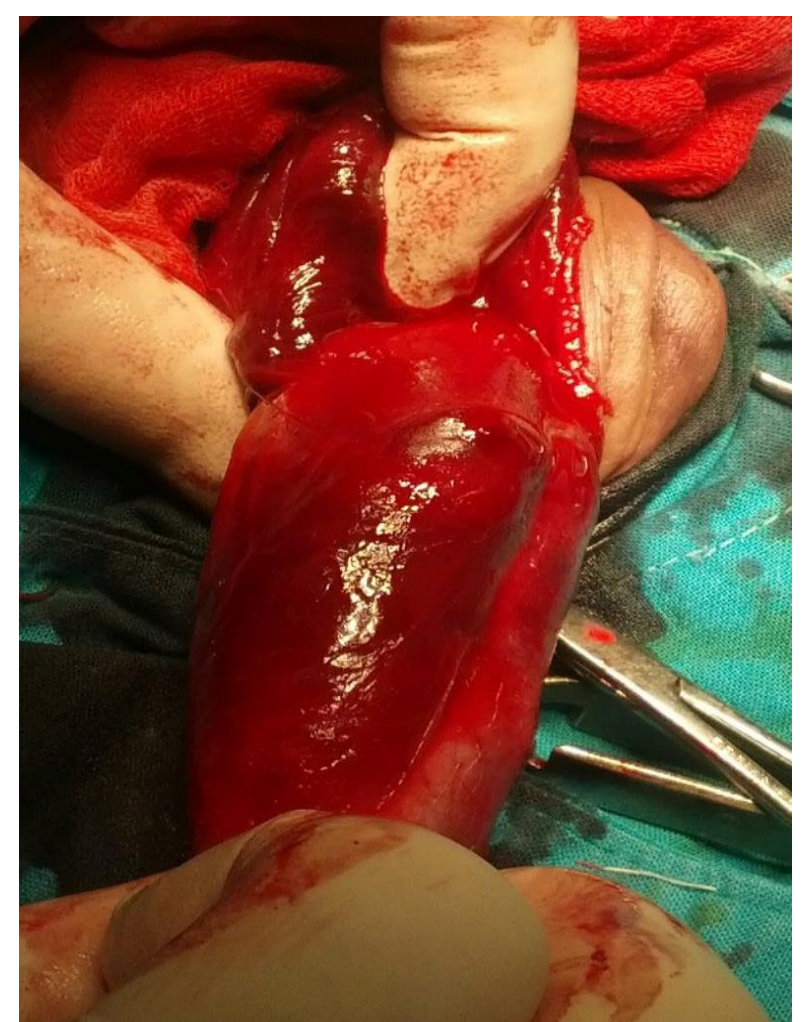

Figure 1 - Haematoma found after degloving

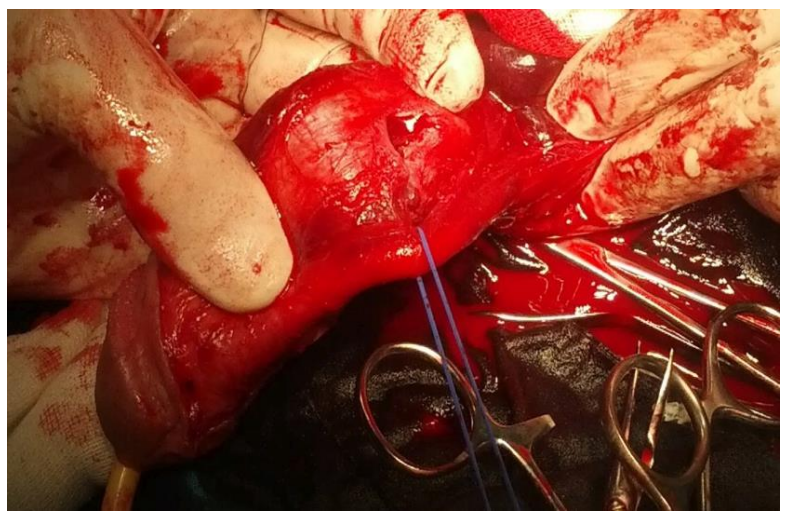

Figure 2 - Isolated urethra and left corpus cavernosum rupture

The patient recovered uneventfully, the Foley catheter was removed on the third day after surgery and he was discharged on the fourth day with no voiding difficulties. The patient regained normal erectile function at two weeks, two days after cessation of erection suppression drugs. The follow-up at 30 days showed no deformity and no pain during erection 


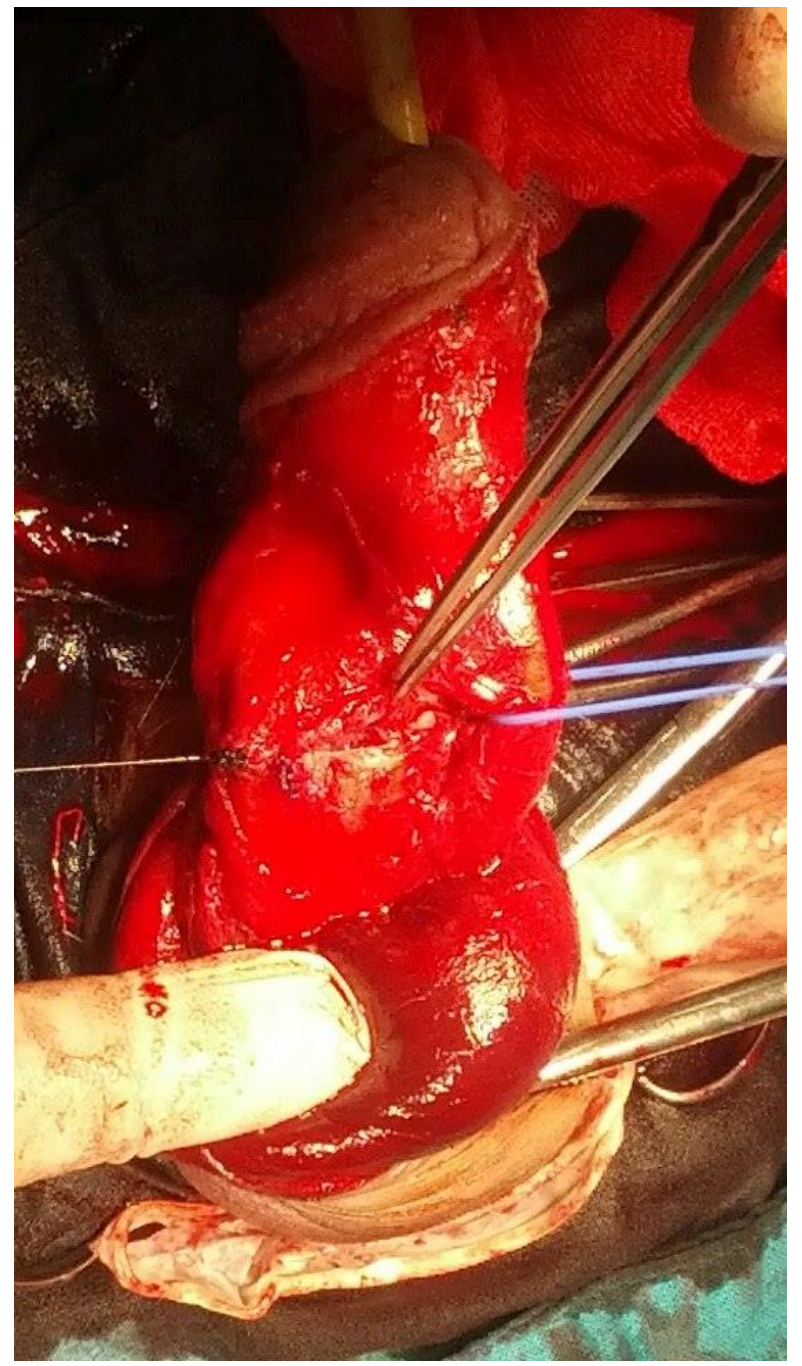

Figure 3 - Intraoperative view: a loop of absorbable suture isolates the right corpus cavernosum defect

\section{Discussion}

Many patients with penile fracture do not seek medical attention due to lack of guidance or shame, therefore penile fractures are one of the most underreported emergency condition in urology. In the vast majority of cases, a penile fracture is caused by trauma during vigorous sexual intercourse (the most common cause in the Western World), when the erect penis hits the perineum or the symphysis pubis. Other causes, such as masturbation, direct blow, and rolling over in bed are less
common.'Taqaandan', a method of forced bending to achieve detumescence, is the most frequent source of penile fracture in the Middle-East [4].

It has been suggested by some authors that structural anomalies in the tunica albuginea could alter its mechanical properties and make rupture possible at intracavernosal pressures inferior to the ones usually necessary for it to snap in patients with a healthy albuginea. These structural anomalies are thought to be caused by repeated previous trauma to the tunica. This theory has been suggested by the detection of fibrosclerosis and cellular infiltrates on the pathology report of the ruptured tunica albuginea $[5,6]$.

The clinical presentation in penile fractures includes a popping sound followed by severe pain, detumescence, and swelling which may extend to the lower abdominal wall [1]. Hematuria may occur when the penile urethra is affected, but the absence of it does not exclude an injury of the urethra and this is why some authors recommended performing a retrograde urethrography on every patient with a clinical presentation suggestive of penile fracture [2]. Other imaging studies that may be used in the diagnosis of penile fractures are ultrasound examination and magnetic resonance imaging. Ultrasound examination can detect a tear of the tunica albuginea in the majority of cases, it is cheap and fast, so it is regarded as the initial imaging method [7]. Cavernosography is useful for a specific diagnosis but it is not done routinely. The technique involves an injection of 15 to $70 \mathrm{~mL}$ solution of $25 \%$ to $50 \%$ nonionic contrast dye (diatrizoate meglumine and diatrizoate sodium are recommended) directly into the uninvolved corpora under live fluoroscopy until both corpora are filled and tumescent changes are noticed. Anteroposterior and oblique radiographs are performed over several time intervals to detect any extravasation from the corpora cavernosa [2].

Eke N. published in the British Journal of Surgery in 2002 one of the most extensive 
reviews regarding penile fractures, analyzing 1331 cases between 1935 and 2001. The main clinical features identified were severe penile pain, swelling, deformation, ecchymosis, and voiding difficulties. Also, the urethral rupture (usually partial) is a commonly associated injury. The long-term complications of penile fracture - especially when the surgical treatment is delayed or avoided - consist of urethral fistula and erectile dysfunction [8].

The condition has mainly a clinical diagnosis, although in the absence of typical signs it can pose a real diagnostic challenge. Therefore, thorough physical examination and patient's history are essential in the diagnostic process.

The gold standard surgical technique for the treatment and repair of penile fractures was described for the first time in 1936 by Fetter and Gartman, reducing the complications of the early conservative treatment (urethral catheterization, antibiotic treatment, antiinflammatory and erection suppressing therapy and ice applications) $[9,10]$.

The differential diagnosis of penile fractures may include vascular lesions of the penis - rupture of the dorsal artery or vein with a similar presentation [5].

Distal degloving represents the gold standard incision for penile fracture surgery because of the great exposure of tunica albuginea and good esthetic results. This approach allows maximal access to both corpora cavernosa and corpus spongiosum, allowing repair of any concomitant urethral tear. Other used approaches are through midline penoscrotal, lateral, inguinoscrotal, and suprapubic incisions. Regardless of the incision performed, it is highly recommended to make a limited circumcision at the end of the procedure. This will prevent postoperative complications such as phimosis and distal skin necrosis [11].

One particular aspect of this case is the bilaterality of the corporeal rupture. This finding is encountered in only $2-10 \%$ of cases [6]. Several reports have confirmed that patients with bilateral corporal rupture should be carefully investigated for associated urethral rupture, which is usually encountered in these cases [12-14]. This case had no urethral lesions despite the ventral location of the corporeal lacerations, as urethrography confirmed. The bilateral involvement of the corpora cavernosa associated with the lack of urethral lesions makes this a rare clinical presentation.

\section{Conclusion}

Penile fracture is a rare urological trauma, but it is largely underreported or medical treatment is unjustifiably postponed leading to long-term sequelae. Diagnosis is usually straightforward, as the clinical findings are highly suggestive for penile fractures, but certain atypical cases may require complex imaging investigations. The presented case pleads for careful clinical examination and surgical exploration to detect bilateral involvement and potential urethral tear.

\section{References}

[1] A. A. Majzoub, O. Canguven, and T. A. Raidh, 'Alteration in the etiology of penile fracture in the Middle East and Central Asia regions in the last decade; a literature review', Urol. Ann., vol. 7 , no. 3, pp. 284-288, 2015.

[2] G. S. Jack, I. Garraway, R. Reznichek, and J. Rajfer, 'Current treatment options for penile fractures', Rev. Urol., vol. 6, no. 3, pp. 114-120, 2004.

[3] M. Mirzazadeh, M. Fallahkarkan, and J. Hosseini, 'Penile fracture epidemiology, diagnosis and management in Iran: a narrative review', Transl. Androl. Urol., vol. 6, no. 2, pp. 158-166, Apr. 2017.

[4] J. Zargooshi, 'Penile fracture in Kermanshah, Iran: report of 172 cases', J. Urol., vol. 164, no. 2, pp. 364-366, Aug. 2000.

[5] R. S. Mahapatra, A. K. Kundu, and D. K. Pal, 'Penile Fracture: Our Experience in a Tertiary Care Hospital', World J. Mens Health, vol. 33, no. 2, pp. 95-102, Aug. 2015. 
[6] N. A. Hoag, K. Hennessey, and A. So, 'Penile fracture with bilateral corporeal rupture and complete urethral disruption: case report and literature review', Can. Urol. Assoc. J. J. Assoc. Urol. Can., vol. 5, no. 2, pp. E23-26, Apr. 2011.

[7] N. Gupta et al., 'Penile fracture: role of ultrasound', Transl. Androl. Urol., vol. 6, no. 3, pp. 580-584, Jun. 2017.

[8] N. Eke, 'Fracture of the penis', Br. J. Surg., vol. 89, no. 5, pp. 555-565, May 2002.

[9] T. R. Fetter and E. Gartman, 'Traumatic rupture of penis: Case report', Am. J. Surg., vol. 32, no. 2, pp. 371-380, May 1936.

[10] D. J. Summerton, A. Campbell, S. Minhas, and D. J. Ralph, 'Reconstructive surgery in penile trauma and cancer', Nat. Clin. Pract. Urol., vol. 2, no. 8, pp. 391-397, Aug. 2005.
[11] F. A. Gulmi, 'NYU Case of the Month, Early February 2018', p. 2.

[12] J. Kowalczyk, A. Athens, and A. Grimaldi, 'Penile fracture: an unusual presentation with lacerations of bilateral corpora cavernosa and partial disruption of the urethra', Urology, vol. 44, no. 4, pp. 599-600; discussion 600-601, Oct. 1994. [13] J. Cumming and J. D. Jenkins, 'Fracture of the corpora cavernosa and urethral rupture during sexual intercourse', Br. J. Urol., vol. 67, no. 3, p. 327, Mar. 1991

[14] A. F. Fergany, K. W. Angermeier, and D. K. Montague, 'Review of cleveland clinic experience with penile fracture', Urology, vol. 54, no. 2, pp. 352-355, Aug. 1999. 